\title{
Composición quimica y actividad antibacteriana del aceite esencial de Ambrosia peruviana Willd. de los llanos venezolanos
}

\author{
Chemical composition and antibacterial activity of the essential oil of \\ Ambrosia peruviana Willd. from Venezuelan plains
}

\author{
Carlos A. Yánez C. ${ }^{4}$, Nurby Rios ${ }^{1}$, Flor Mora ${ }^{1}$, Luis Rojas², Tulia Diaz ${ }^{3}$, Judith Velasco ${ }^{3}$, \\ Nahile Rios ${ }^{4}$ y Pablo Melendez ${ }^{1}$
}

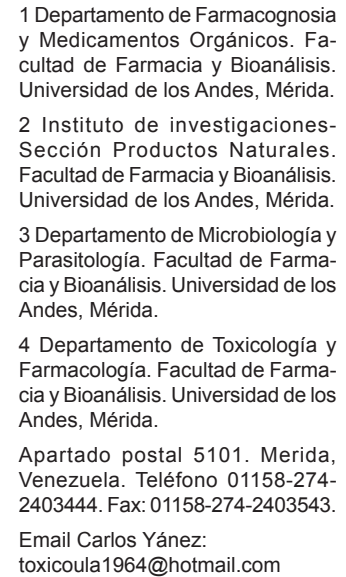

toxicoula1964@hotmail.com

\begin{abstract}
Resumen
En Venezuela actualmente se están explorando nuevas fuentes de agentes antibacterianos de origen natural, debido al aumento de la resistencia bacteriana, entre ellos los aceites esenciales derivados de plantas. Por tal razón en el presente estudio se determinó la composición química del aceite esencial obtenido de las hojas de Ambrosia peruviana Willd recolectada en Guasdualito, Estado Apure, Venezuela. Los compuestos volátiles se aislaron por hidrodestilación en una trampa de Clevenger y posteriormente se realizó el análisis cualitativocuantitativo a través de cromatografía de gases acoplada a espectrometría de masas (GC/MS), en un equipo HP GC-MS System, modelo 5973, encontrando como compuestos mayoritarios al gamma-curcumeno $(23,99 \%)$, seguido de ar-curcumeno (14,08\%), acetato de bornilo (10,35\%), camfor $(5,03 \%)$ y epóxido de oximene $(4,79 \%)$. La actividad antibacteriana del aceite esencial realizada por el método de difusión en agar con discos frente a Staphylococcus aureus, Enterococcus faecalis, Escherichia coli, Klebsiella pneumoniae, Salmonella Typhi y Pseudomonas aeruginosa, mostró actividad contra S. aureus, E. faecalis, E. coli, y S. Typhi, con valores de CIM de 350-500 $\mu \mathrm{g} / \mathrm{mL}$. Esta investigación es el primer reporte de actividad antibacteriana de A. peruviana.
\end{abstract}

Palabras claves: Ambrosia peruviana, aceite esencial, actividad antibacteriana.

\section{Abstract}

In Venezuela, are currently exploring new sources of natural antibacterial agents, due to increased bacterial resistance, including essential oils derived from plants. For this reason in the present study we determined the chemical composition of essential oil obtained from leaves collected on Ambrosia peruviana Willd Guasdualito, Apure State, Venezuela. The volatile compounds were isolated by hydrodistillation in a Clevenger trap and then subjected to qualitative analysis and quantitative by gas chromatography-mass spectrometry (GC / MS) on an HP GC-MS System, model 5973, finding as the major compound gamma-curcumeno $(23.99 \%)$ followed by curcumeno-ar $(14.08 \%)$, bornyl acetate $(10.35 \%)$, camphor $(5.03 \%)$ and epoxide oximene $(4.79 \%)$. The antibacterial activity of essential oil by the agar diffusion method with discs against Staphylococcus aureus, Enterococcus faecalis, Escherichia coli, Klebsiella pneumoniae, Salmonella typhi and Pseudomonas aeruginosa showed activity against S. aureus, E. faecalis, E. coli and S. Typhi, with MIC values of 350-500 micrograms/ $\mathrm{mL}$. This research represents the first report of antibacterial activity of $A$. peruviana.

Keywords: Ambrosia peruviana, essential oil, antibacterial activity.

\section{Introducción}

En la flora de Venezuela, la familia Asteraceae (Compositae) es la segunda familia con mayor riqueza de especies en la división Magnoliophyta; se reconocen 204 géneros y 784 especies nativas o naturalizadas, especialmente en las regiones Andino-Cordillera Costera y Alta Guayana; crecen en sitios con temperaturas bajas, por esta razón son pocas las especies en la región Costera y de los Llanos (Lasser 1964, Hokecheo et al. 2008).

El género Ambrosia ha sido estudiada como antioxidante por los flavonoides y glicósidos que contienen (Zoran et al. 2008), y que son utilizados en el tratamiento de infecciones por Schistosoma mansoni (Abadome et al. 1994), antiepiléptico (Buznego et al. 1998, Buznego \& Pérez 2004), y como antiinflamatorio por la capacidad del compuesto cumain de inhibir la producción de óxido nítrico, importante mediador en los procesos inflamatorios (Lastra et al. 2004).

Ambrosia peruviana Willd. (Asteraceae) es una hierba de $4-$ $8 \mathrm{~cm}$ de largo distribuida desde México hasta la parte tropical de América del sur; conocida vulgarmente con el nombre de altamisa, artemisa, altamiz, alcanfor (Cuba), Ambrosia silvestre, Maki (Bolivia). En Venezuela se encuentra en los estados Sucre, Distrito Federal, Apure, Bolívar, Falcón, Mérida, Miranda, Táchira, Trujillo y Delta Amacuro (Badillo 2001, Lasser 1964).
Ambrosia peruviana se emplea como hipotensor, antiespasmódico, depurativo, diaforético y en trastornos menstruales (Hernández et al. 2002, Lans 2007, Zapata et al. 2010). En Venezuela $A$. peruviana es utilizada para dolor del cuerpo, fiebre, dolor de cabeza, hipotensión, ulceras, manchas de la piel, varices, cicatrices, trastornos menstruales (Gil et al. 2006).

Aunque el género Ambrosia ha sido ampliamente estudiado fitoquímicamente, son escasos los trabajos relacionados con la composición química de sus aceites esenciales. Chalchat et al. (2004) identificaron 51 compuestos en el aceite esencial obtenido de Ambrosia artemisiifolia recolectada en Belgrado (República de Serbia) sus principales componentes fueron: Dgermacrene $(24,1 \%)$, limoneno $(16,83 \%)$, alfa-pineno $(8,0 \%)$ y mircineno $(7,4 \%)$, y observaron importante actividad bactericida y fungicida. La composición química del aceite esencial de Ambrosia trifida de China representa acetato de bornilo $(15,5 \%)$, borneol $(8,5 \%)$, óxido de cariofileno $(8,3 \%)$, alfapineno $(8,0 \%)$ atribuyendo a estos la actividad antibacteriana (Wang et al. 2006).

El presente trabajo tiene como objetivo identificar los componentes del aceite esencial de $A$. peruviana recolectada en Venezuela y estudiar su actividad antibacteriana, por esta razón esta investigación resulta un gran aporte a la química de productos naturales. 


\section{Material y métodos}

Material vegetal.- Hojas de $A$. peruviana fueron recolectadas en mayo 2010 en Guasdualito (07 14 '49"N 7043'1"W, 125 $\mathrm{m}$ de altitud), Estado Apure, Venezuela. Una muestra fue depositado (voucher número NR011) en el Herbario MERF de la Facultad de Farmacia y Bioanálisis, Universidad de los Andes, Mérida, Venezuela.

Obtención del aceite esencial.- Hojas frescas (1305 g) de $A$. peruviana se trituraron y sometieron a destilación por arrastre con vapor de agua (6 horas), empleando una trampa de tipo Clevenger (Rojas et al. 1999). Un mililitro del aceite esencial color amarillo (0,09\% rendimiento) fue obtenido. El aceite se conservó a $-4^{\circ} \mathrm{C}$ hasta su uso para ensayos biológicos.

Análisis de composición química.- Los componentes volátiles del aceite esencial fueron analizados por cromatografía de gases utilizando un cromatógrafo Perkin-Elmer. La identificación de cada uno de los compuestos se realizó por GC-MS con un equipo Hewlett Packard Modelo 5973, equipado con una columna HP-5MS de $30 \mathrm{~m}$ de largo x 0,25 mm de diámetro $\mathrm{x}$ $0,25 \mu \mathrm{m}$ de grosor de la película. Temperatura de inyector 230 ${ }^{\circ} \mathrm{C}$, temperatura del cuádruplo $150^{\circ} \mathrm{C}$, gas transportador Helio a una velocidad lineal de $34 \mathrm{~m} / \mathrm{s}$; energía de ionización $70 \mathrm{eV}$; rango de scan de 40-50 amu; 3,9 scan/s. Volumen de inyección $1 \mu \mathrm{L}$ de una solución diluida al $2 \%$ en éter dietílico. La identificación de los compuestos fue basada en la base de datos Wiley MS Data Library y NIST 05, los índices de Kovats se compararon con valores disponibles en la literatura ( $6^{a}$ edición) (Adams 2007).

Actividad antibacteriana.- La actividad antibacteriana fue evaluada de acuerdo al método de difusión en agar con discos descrita por Salazar et al. (2007), utilizando las cepas de referencia Staphylococcus aureus ATCC 25923, Enterococcus fecalis

Tabla 1. Composición química del aceite esencial de Ambrosia peruviana $(\%)^{*}$.

\begin{tabular}{clcc}
\hline Pico N $^{\circ}$ & \multicolumn{1}{c}{ Compuestos $^{\mathbf{a}}$} & Área (\%) & Ik $^{\mathbf{b}}$ \\
\hline 1 & sabineno & 0,3 & 976 \\
2 & beta-pineno & 2,0 & 979 \\
3 & cis-epoxi-oximeno & 4,8 & 996 \\
4 & silvestreno & 2,9 & 1031 \\
5 & cineol-1,8 & 0,4 & 1035 \\
6 & fenchona & 0,6 & 1092 \\
7 & linalol & 1,1 & 1101 \\
8 & camfor & 5,0 & 1152 \\
9 & cis-derbenol & 1,8 & 1169 \\
10 & borneol & 3,6 & 1173 \\
11 & tepinen-4-ol & 0,4 & 1185 \\
12 & Acetato de bornilo & 10,4 & 1277 \\
13 & beta-cariofileno & 1,4 & 1429 \\
14 & bergamoteno-alfa- & 1,3 & 1446 \\
15 & cis & 1,5 & 1468 \\
16 & trans-beta-farneseno & 24,0 & 1493 \\
17 & curcumeno-gamma & 14,1 & 1496 \\
18 & curcumeno-ar & 2,7 & 1521 \\
19 & nerolidol-Z & 0,6 & 1535 \\
20 & cadineno-delta & 1,6 & 1600 \\
21 & junenol & 3,3 & 1612 \\
22 & cubenol & 0,69 & \\
\hline
\end{tabular}

a Lista de Compuestos en orden de elución con dos columnas: AT-5 (apolar)

b Índice de Kovats calculados en relación a $n$-alcanos (C8-C24)
ATCC 29212, Escherichia coli ATCC 25922, Klebsiella pneumoniae ATCC 23357, Salmonella Typhi CDC57 y Pseudomona aeruginosa ATCC 27853 proporcionadas por el Departamento de Microbiología y Parasitología de la Facultad de Farmacia y Bioanálisis de la Universidad de los Andes.

En los organismos que mostraron zona de inhibición se determinado la concentración inhibitoria mínima (CIM), preparando diluciones del aceite con dimetilsulfóxido a concentraciones de 10 a $600 \mu \mathrm{g} / \mathrm{mL}$, fueron empleados los antibióticos de referencia: Eritromicina ${ }^{\oplus}(150 \mu \mathrm{g})$, Vancomicina ${ }^{\oplus}(30 \mu \mathrm{g})$, Ampicilina-Sulbactam ${ }^{\oplus}(10 \mu \mathrm{g}-10 \mu \mathrm{g})$, Aztreonam ${ }^{\oplus}(30 \mu \mathrm{g})$, Ciprofloxacina $^{\oplus}(30 \mu \mathrm{g})$ y Ceftazidima ${ }^{\circledR}(30 \mu \mathrm{g})$. Los ensayos se realizaron por duplicado.

\section{Resultados y discusión \\ Composición química del aceite}

Fueron identificados 22 compuestos (84,5\%) en el aceite esencial obtenido de $A$. peruviana (Tabla 1 ); los principales componentes encontrados fueron el gamma-curcumeno $(23,99 \%)$, ar-curcumeno (14,08\%), acetato de bornilo (10,35\%), camfor $(5,03 \%)$ y epóxido de oximeno $(4,79 \%)$.

\section{Actividad antibacteriana}

La actividad antibacteriana del aceite esencial realizada por el método de difusión en agar con discos frente a bacterias gram positivas y negativas, mostró actividad contra $S$. aureus, E. faecalis, E. coli, y S. typhi, con valores de CIM de 350-500 $\mu \mathrm{g} / \mathrm{mL}$ (Tabla 2). Los resultados obtenidos en las bacterias gram positivas coincidieron con los registrados por Wang et al. (2006) y Chalchat et al. (2004), sin embargo estos autores reportaron actividad antibacteriana en $K$. pneumoneae y $P$. aeruginosa no observadas en nuestra investigación.

El método de difusión en agar es uno de los métodos frecuentemente empleados para ensayar la actividad antibacteriana de aceites esenciales, sin embargo se han observado divergencias relacionadas con la actividad individual de los componentes del aceite, por ejemplo el 1,8-cineol obtenido del aceite de las hojas de Té no muestra halos de inhibición pero si valores de MIC bajos (Kalemba et al. 2003).

\section{Conclusión}

De acuerdo con los resultados obtenidos en este trabajo se concluye que los compuestos químicos que conforman el aceite esencial de Ambrosia peruviana de los Llanos venezolanos, difieren de otros aceites esenciales obtenidos del género Ambrosia. Sin embargo, los componentes acetato de bornilo, camfor, y borneol se encuentran en $A$. trifida, $A$. artemisiifolia y $A$. peruviana. Los compuestos más importantes del aceite esencial obtenido de Ambrosia peruviana Willd. fueron gamma-curcumeno (23,99\%), ar-curcumeno (14,08\%), acetato de bornilo (10,35\%), camfor $(5,03 \%)$ y cis-epóxido-oximeno $(4,79 \%)$, los que podrían ser responsables de la actividad antibacteriana.

\section{Literatura citada}

Abadome F., G. Geerts, V. Kumar. 1994. Evaluation of the activity of Ambrosia maritima L. against Schistosoma mansoni infection in mice. Journal of Ethnopharmacology. 44:195-198.

Adams R. 2007. Identification of essential oil components by gas chromatograpy/mass spectroscopy, 4th Edition. Illinois USA: Allured Publishing Corporation, Carol Stream, Ill, p. 804. 
Tabla 2. Actividad antibacteriana del aceite esencial de Ambrosia peruviana contra cepas de Referencia Internacional.

\begin{tabular}{|c|c|c|c|c|c|c|c|c|}
\hline \multirow[b]{3}{*}{ Microrganismos } & \multicolumn{8}{|c|}{ Zona de inhibición } \\
\hline & \multirow[b]{2}{*}{ Aceite esencial } & \multicolumn{6}{|c|}{ Antibióticos de referencia } & \multirow{2}{*}{$\begin{array}{c}\text { CIM } \\
(\mu \mathrm{g} / \mathrm{mL})\end{array}$} \\
\hline & & $\mathrm{E}$ & VA & SAM & AZT & CIP & CAZ & \\
\hline Staphylococcus aureus ATCC (25923) & $8^{*}$ & $30^{*}$ & & & & & & 400 \\
\hline Enterococcus faecalis ATCC (29212) & $11^{*}$ & & $22^{*}$ & & & & & 500 \\
\hline Escherichia coli ATCC (25922) & $7^{*}$ & & & $25^{*}$ & & & & 500 \\
\hline Klebsiella pneumoniae ATCC (23357) & NA & & & & $33^{*}$ & & & NP \\
\hline Salmonella typhi CDC 57 & $8^{*}$ & & & & & $45^{*}$ & & 350 \\
\hline Pseudomonas aeruginosa ATCC (27853) & NA & & & & & & $31^{*}$ & NP \\
\hline
\end{tabular}

${ }^{*} \mathrm{~mm}$ de los halos de inhibición (discos $6 \mathrm{~mm}$ de diámetro) promedio de dos ensayos consecutivos; NA: no activo; NP: no probado; E: Eritromicina® (150 $\mu$ ), VA: Vancomicina® (30 $\mu \mathrm{g}$ ) SAM: Ampicilina-Sulbactam $₫(10 \mu \mathrm{g} / 10 \mu \mathrm{g})$, AZT: Aztreonam $₫ 30 \mu \mathrm{g}$, CIP: Ciprofloxacina $30 \mu \mathrm{g}$, CAZ: Ceftazidima $30 \mu \mathrm{g}$. CIM. Concentración Inhibitoria mínima, rango de concentración $10-600 \mu \mathrm{g} / \mathrm{mL}$.

Badillo V.\& I. Lapp. 2001. Lista Actualizada de Compuestas en Venezuela. Ernstia. 11:147-215.

Buznego M., M. Llanio, M. Fernández, N. Alonso, M. Acevedo, H. Pérez. 1998. Perfil neurológico de la Ambrosia paniculata (Willd) O.E Schulz (Artemisa). Rev Cubana Plant Med. 3:45-46.

Buznego M., H. Pérez. 2004. Acute effect of an extract of Ambrosia paniculata (Willd.) O. E. Schultz (mugwort) in several models of experimental epilepsy. Epilepsy \& Behavior. 5:847-851.

Chalchat C., A. Maksimović, D. Petrović, S. Gorunović, S. Đorđević, M. Mraović. 2004. Chemical composition and antimicrobial activity of Ambrosia artemisiifolia L. essential oil. Journal of Essential Oil Research. 16:270-273.

Gil R., J. Carmona, M. Rodríguez. 2006. Estudio etnobotánico de especies toxicas, ornamentales y medicinales de uso popular presentes en el jardín de plantas medicinales. Boletín antropológico. 24:463-481.

Hernández J., H. Valero, R. Gil. 2002. 23 especies vegetales medicinales de uso frecuente en la población de Tabay. Revista de la Facultad de Farmacia. 44:51-57.

Hokecheo O., P. Berry, O. Huber. 2008. Nuevo Catalogo de la Flora Vascular de Venezuela. Fundación Instituto Botánico de Venezuela Dr. Tobias Lasser. Caracas. Venezuela.

Kalemba D. \& A. Kunicka. 2003. Antibacterial and antifungal properties of essential oils. Current Medicinal Chemistry. 10:813-829.

Lans C. 2007. Ethnomedicines used in Trinidad and Tobago for reproductive problems. Journal of Ethnobiology and Ethnomedicine. 3:1-13

Lasser T. 1964. Flora de Venezuela Vol X. Parte Primera. Instituto Botánico Dirección de Recursos Naturales Renovables. Ministerio de Agricultura y Cría. Caracas, Venezuela. 244:475-478.
Lastra A., T. Ramírez, L. Salazar, M. Martínez, F. Trujillo. 2004. The ambrosanolide cumain inhibits macrophage nitric oxide synthesis: some structural considerations. Journal Ethnofarmacology. 95:221-227.

Rojas L. 1994. Trabajo Especial de Grado ULA. Facultad de Farmacia. Postgrado en Química de Medicamentos.

Salazar E., B. Nieves, M. Ruiz, M. Araque, E. Velazco, J. Vila. 2007. Molecular Epidemiology and Characterization of Resistance Mechanisms to various Antimicrobial Agents in Acinetobacter baumanni. Medical Science Monitor. 13:89-94

Wang P., C. Hua, C. Xian. 2006. Chemical composition and antibacterial activity of the essential oil from Ambrosia trifida L. Molecules, 11:549-555.

Zapata B., C. Durán, E. Stashenko, L. Betancur, A. Mesa. 2010. Actividad antimicótica y citotóxica de aceites esenciales de plantas de la familia Asteraceae. Revista Iberoamericana de Micología. 27:101-103.

Zoran M. 2008. In vitro antioxidant activity of ragweed (Ambrosia artemisiifolia L., Asteraceae) herb. Industrial Crops and Products. 28:356-336. 
